\title{
Constraints on the Lyman continuum radiation from galaxies: First results with FUSE on Mrk 54*
}

\author{
J.-M. Deharveng ${ }^{1}$, V. Buat ${ }^{1}$, V. Le Brun ${ }^{1}$, B. Milliard ${ }^{1}$, D. Kunth ${ }^{2}$, J. M. Shull ${ }^{3}$, and C. Gry ${ }^{1,4}$ \\ 1 Laboratoire d'Astrophysique de Marseille, Traverse du Siphon, Les Trois Lucs, BP 8, 13376 Marseille Cedex 12, \\ France \\ 2 Institut d'Astrophysique de Paris, 98bis boulevard Arago, 75014 Paris, France \\ 3 Center for Astrophysics and Space Astronomy, Department of Astrophysical and Planetary Sciences, \\ University of Colorado, Boulder, CO 80309, USA \\ 4 ISO Data Center, ESA Astrophysics Division, PO Box 50727, 28080 Madrid, Spain
}

Received 16 March 2001 / Accepted 14 June 2001

\begin{abstract}
We present Far Ultraviolet Spectroscopic Explorer observations of the star-forming galaxy Mrk 54 at $z=0.0448$. The Lyman continuum radiation is not detected above the H I absorption edge in our Galaxy. An upper limit is evaluated by comparison with the background measured in regions of the detector adjacent to the observed spectrum. A spectral window of $16 \AA$, reasonably free of additional H i Lyman series line absorption, is used. No correction is needed for molecular hydrogen absorption in our Galaxy but a foreground extinction of $0.29 \mathrm{mag}$ is accounted for. An upper limit of $6.15 \times 10^{-16} \mathrm{erg} \mathrm{cm}^{-2} \mathrm{~s}^{-1} \mathrm{~A}^{-1}$ is obtained for the flux at $\approx 900 \AA$ in the rest frame of Mrk 54. By comparison with the number of ionizing photons derived from the $\mathrm{H} \alpha$ flux, this limit translates into an upper limit of $f_{\text {esc }}<0.062$ for the fraction of Lyman continuum photons that escape the galaxy without being absorbed by interstellar material. This limit compares with the limits obtained in three other nearby galaxies and is compatible with the escape fractions predicted by models. The upper limits obtained in nearby galaxies contrasts with the detection of Lyman continuum flux in the composite spectrum of Lyman-break galaxies at $z \approx 3.4$. The difficulties and implications of a comparison are discussed.
\end{abstract}

Key words. galaxies: individual: Mrk 54 - intergalactic medium - galaxies: ISM - galaxies: starburst - ultraviolet: galaxies

\section{Introduction}

It is not yet clear whether hot and massive stars forming in galaxies contribute significantly to the ionizing background radiation in the universe, and how this contribution evolves as a function of redshift (e.g. Bechtold et al. 1987; Miralda-Escudé \& Ostriker 1990; Meiksin \& Madau 1993; Madau \& Shull 1996; Haardt \& Madau 1996; Shull et al. 1999). Specifically, at redshifts $z>3$, the early formation of galaxies is expected to compensate for the decline in the quasar contribution and to play a role in the re-ionization of the IGM (e.g. Madau et al. 1999).

Direct observations of galaxies below the Lyman break have been scarce so far. With the Hopkins Ultraviolet Telescope (HUT), Leitherer et al. (1995),

Send offprint requests to: J.-M. Deharveng, e-mail: jean-michel.deharveng@astrsp-mrs.fr

* Based on observations made with the NASA-CNES-CSA Far Ultraviolet Spectroscopic Explorer. FUSE is operated for NASA by the Johns Hopkins University under NASA contract NAS5-32985.
Hurwitz et al. (1997) obtained upper limits on the Lyman continuum ( $\mathrm{LyC}$ ) radiation in four nearby star-forming galaxies. By comparison with the $\mathrm{H} \alpha$ emission these data were interpreted in terms of limits on the LyC escape fraction, a parameter giving the fraction of hydrogen-ionizing photons effectively released into the IGM. Recently Steidel et al. (2001) reported the detection of the LyC radiation in a composite spectrum of Lyman break galaxies at $z \sim 3.4$ that was also interpreted in terms of LyC escape fraction but by comparison with the $(1500 \AA) \mathrm{UV}$ continuum. A preliminary account of the Space Telescope Imaging Spectrograph (STIS) observations of galaxies in the Hubble Deep Field (HDF), with implications for the LyC escape fraction, has been presented by Ferguson (2001) while this paper was nearing completion.

In the absence of a large number of observations that would directly provide the LyC luminosity function and the LyC luminosity density of galaxies, the LyC escape fraction is seen as a crucial parameter. Combined with the $\mathrm{H} \alpha$ or UV luminosity densities of galaxies or with the stellar ionizing radiation calculated from evolutionary 
synthesis models (e.g. Bruzual \& Charlot 1993; Leitherer et al. 1999) or from the rate of chemical enrichment in the universe (Cowie 1988; Songaila et al. 1990; Madau \& Shull 1996), the LyC escape fraction provides the amount of ionizing radiation effectively released into the IGM by the galaxies. As this parameter encapsulates a number of complex and random factors it is probably highly variable from galaxy to galaxy; its full understanding and sound utilisation would also require a large number of observations.

The Far Ultraviolet Spectroscopic Explorer (FUSE) has recently opened again an access into the far ultraviolet down to $905 \AA$, allowing the possibility to observe the LyC radiation of low redshift galaxies above the $912 \AA$ Lyman limit of $\mathrm{H}$ I photoelectric absorption in our Galaxy. As shown by the re-analysis of HUT data by Hurwitz et al. (1997), getting rid of photoelectric absorption in our Galaxy above the Lyman edge is not enough and gasphase absorption in our Galaxy, essentially from convergent H I Lyman series and the Lyman and Werner bands of molecular hydrogen, has to be accounted for, in addition to dust extinction. It is an area where the high spectral resolution of FUSE can help, even though the increased background contribution per wavelength unit makes the detection of faint continua more difficult than at low resolution. In the following, we report the FUSE observations of a star-forming galaxy Mrk 54, at a redshift $z=0.0448$ that places the Lyman break at $952.5 \AA$ above the Galactic Ly $\delta$ absorption feature, reasonably beyond the convergent H I Lyman series.

\section{Observations and data processing}

The observations of Mrk 54 (GI program A052) were obtained on Feb. 19-20, 2000 in the time-tagged photon address mode with the object in the large $\left(30^{\prime \prime} \times 30^{\prime \prime}\right)$ aperture. The observations were split into 15 exposures between occultation periods and passages through the South Atlantic Anomaly, and generally extended over spacecraft night and day. The total duration was $27502 \mathrm{~s}$. Details of the FUSE instrument and on-orbit performance have been given by Moos et al. (2000) and Sahnow et al. (2000).

The summed and calibrated spectrum of Mrk 54 is consistent at long wavelength with the IUE observations (Kinney et al. 1993) and reaches a maximum flux of about 3. $\times 10^{-14} \mathrm{erg} \mathrm{cm}^{-2} \mathrm{~s}^{-1} \mathrm{~A}^{-1}$ at $\sim 1100 \AA$. At wavelengths shorter than $1000 \AA$ the flux decreases rapidly and below $960 \AA$ the signal, if any, becomes extremely weak (Fig. 1); actually it drops to below zero between the Lyman-series airglow lines with the uniform background correction of 1 count $\mathrm{cm}^{-2} \mathrm{~s}^{-1}$ applied by the standard calibration pipeline. The faint level between the redshifted Lyman break of the galaxy $911.7(1+0.0448)=952.5 \AA$ and about $960 \AA$ is probably due to the accumulation of Lyman series absorption lines in the galaxy itself. These lines are resolved from $\operatorname{Ly} \beta$ to $\operatorname{Ly} 7$ ( $\mathrm{Ly} \gamma$ and $\operatorname{Ly} \beta$ are not shown in Fig. 1).

For the purpose of discussing the $\mathrm{LyC}$ radiation of the galaxy Mrk 54 we have concentrated our analysis on the

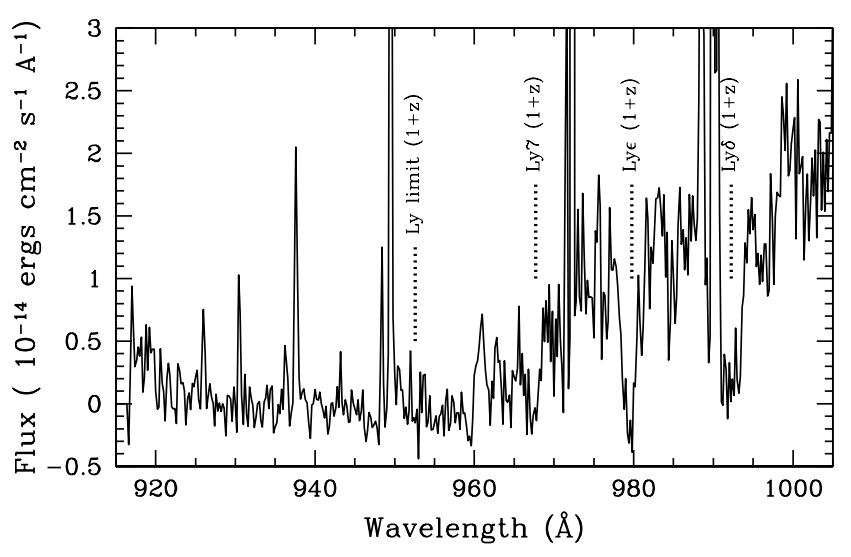

Fig. 1. FUSE spectrum of Mrk 54 resulting from the coaddition of $15 \mathrm{SiC} 2 \mathrm{~A}$ spectra for a total exposure time of $27502 \mathrm{~s}$ and at $0.2 \AA$ linear rebinning. The Lyman series absorption lines resolved in Mrk 54 are marked, except for Ly6 confused with the Ly $\gamma$ airglow line at $972.5 \AA$.

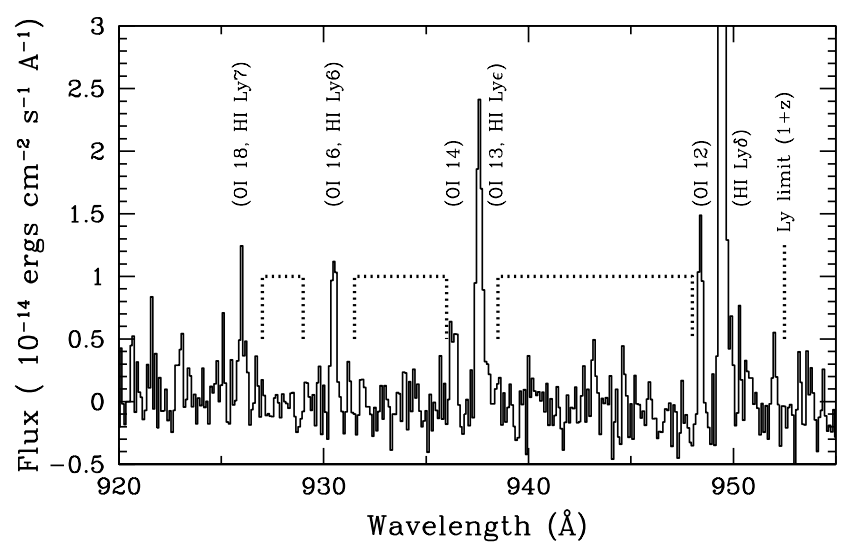

Fig. 2. Details of the previous spectrum (rebinned at $0.1 \AA$ ), showing (dotted line) the three spectral windows (16 $\AA$ total) used for obtaining an upper limit to the LyC of Mrk 54. Tentative identifications of the airglow lines based on Feldman et al. (2000) are indicated.

$\mathrm{SiC}$ channels and specifically the $\mathrm{SiC} 1 \mathrm{~B}$ and $\mathrm{SiC} 2 \mathrm{~A}$ spectra. We have screened the raw time-tag data of segments $1 \mathrm{~B}$ and $2 \mathrm{~A}$ for the presence of so-called bursts, using for each of the 15 exposures both the images of the events and the time series of the total number of counts. The latter has shown some increase of the count rate at the end of a few exposures: as these features were not typical burst events we decided not to remove them from the time-tag data (even for the most significant in exposure 13). We made no attempt to isolate night-only data.

In the absence of any clear signal from the redshifted LyC of the galaxy (shortward of $952.5 \AA$ ) we have tried to place an upper limit to this flux. A first step is to determine spectral windows that are free from additional absorption by neutral hydrogen in our Galaxy (Hurwitz et al. 1997). As discussed by these latter authors, it is a complex issue given the lack of information regarding low column density clouds at velocities departing significantly from that of the bulk of the HI. The examples given by 
Hurwitz et al. (1997) and by Lockman \& Savage (1995) as well as the examination of the list of high-velocity clouds of Stark et al. (1992) lead us to conclude that removing $\pm 200 \mathrm{~km} \mathrm{~s}^{-1}$ on either side of the Lyman series absorption lines is probably safe enough. This selection removes the $\mathrm{H}$ I nightglow lines located right at the rest wavelengths of the potential absorption lines. A comparison of our spectrum with the reference airglow spectrum of Feldman et al. (2000) shows that we should also avoid a few O I lines close to $\mathrm{HI}$ lines and discard a slightly larger wavelength domain shortward of each Lyman series absorption line. This corresponds to a velocity of $\approx-500 \mathrm{~km} \mathrm{~s}^{-1}$, making our previous limit even safer on the side of negative velocities. With the constraints adopted, it is not possible to extend our study shortward of Ly7 (926.2 $\AA$ ). We practically end up with a window of $16 \AA$, total, split into three domains, 927-929 $\AA, 931.5-936 \AA$ and $938.5-948 \AA$ by the Lyman series lines Ly6 $930.7 \AA$, Ly $\epsilon 937.8 \AA$ and $\mathrm{Ly} \delta$ $949.7 \AA$ (Fig. 2). We have calculated the total raw counts in this window for each of the $15 \mathrm{SiC} 1 \mathrm{~B}$ and $15 \mathrm{SiC} 2 \mathrm{~A}$ extracted spectra.

These raw counts can be compared with those obtained in reference zones, defined in the 15 images of segments $1 \mathrm{~B}$ and $2 \mathrm{~A}$ built from the corresponding raw thag data (Fig. 3). These reference zones, supposed to represent the background at the time of observations, have been searched as close as possible to the detector areas where the sky observations are made. Our search was guided by the examination of profiles integrated over a large number of lines or columns of the co-added (15) images in order to avoid detector edge effects and airglow lines in the smaller apertures. We end up with three areas of respectively 542101,1023701 and 737751 pixels in the 1B segment and 322361, 610151 and 443631 pixels in the 2A segment. In these zones the counts are found to follow Poisson statistics extremely well.

Three features stand out in Fig. 3. First, given their $(1 \sigma)$ error bars the three background measurements are neither significatively different in each exposures nor show systematic variation pattern. This is taken as a good indication that the background is reasonably uniform, at least at the scale and in the zones we are using. In contrast, there are significant variations of the background measurements (departing from purely Poisson statistics) from exposure to exposure that can be understood by differences in observing conditions. Third, the target measurements follow the same trend as the background from exposure to exposure (except may be for exposure 7) and, given their larger error bars, do not appear significantly different from the background measurements.

These findings justify the use of our evaluation of the background in each image to correct the sky measurement. The error bars on the resulting net counts in each exposure combine quadratically the error bar on the sky measurements and those on the background scaled to the surface of the sky measurements; they are dominated by the uncertainties on the sky measurements based on a smaller number of pixels. The error bars of

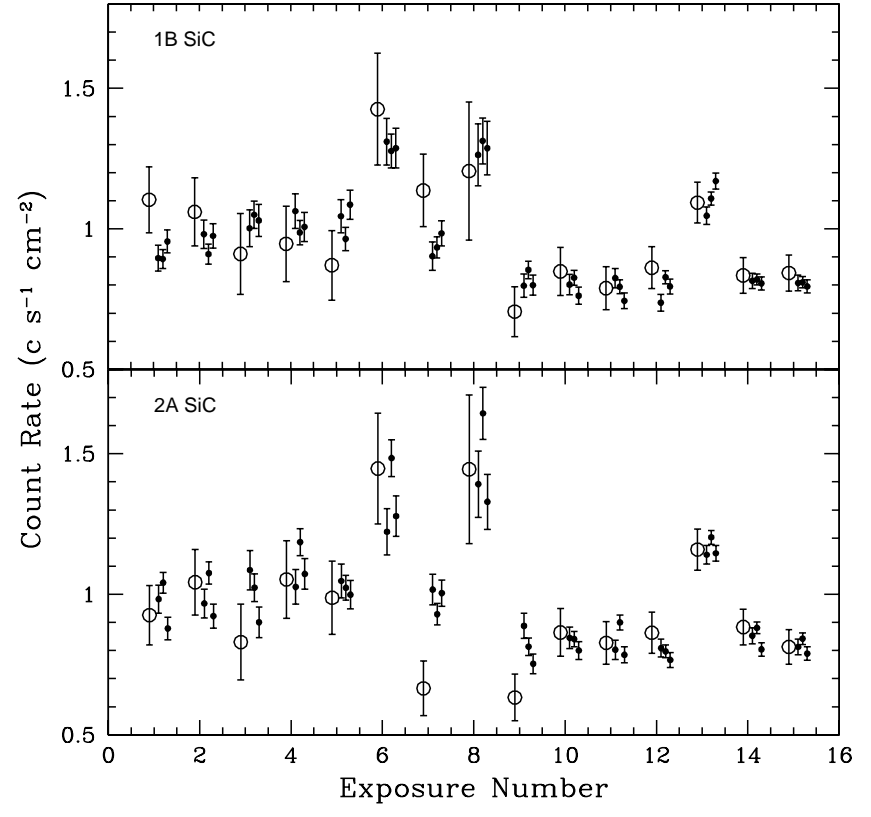

Fig. 3. Comparison of the sky raw counts (open circle) in the $16 \AA$ window defined in the text with the background counts in three reference zones (solid dots). These zones are displayed in the same relative order for each 15 exposures. For the purpose of comparison all counts have been expressed into units of counts $\mathrm{cm}^{-2} \mathrm{~s}^{-1}$ using the respective exposure times, the surface of the extracting windows for the sky counts, the number of pixels and their linear size (respectively $5.98 \times 9.08 \mu \mathrm{m}$ for the $1 \mathrm{~B}$ segment and $5.96 \times 14.75 \mu \mathrm{m}$ for the $2 \mathrm{~A}$ segment) for the background zones. $1 \sigma$ Poisson error bars are given.

each 15 exposures are then combined quadratically and divided by the total exposure time to give the dispersion on the net count rate. We obtain a mean net count rate of $1.32 \times 10^{-3}$ count $\mathrm{s}^{-1}$ with a $1 \sigma$ dispersion of $1.40 \times 10^{-3}$ count s$^{-1}$ and $-1.13 \times 10^{-3}$ count s $^{-1}$ with a $1 \sigma$ dispersion of $1.43 \times 10^{-3}$ count s $^{-1}$ in the $16 \AA$ window of segments $1 \mathrm{~B}$ and $2 \mathrm{~A}$ respectively. The fact that the mean count rates are smaller than the dispersion confirms quantitatively the trends discussed above. Under these conditions the net count rate dispersion can be used to set an upper limit on the flux of Mrk 54 at $\approx 900 \AA$ (restframe). Combining the two segments and converting the counts into flux (using an effective area of the order of $17 \mathrm{~cm}^{2}$ at $\sim 940 \AA$, according to the on-orbit performance reported by Sahnow et al. 2000) we get a $3 \sigma$ upper limit of $4.7 \times 10^{-16} \mathrm{erg} \mathrm{cm}^{-2} \mathrm{~s}^{-1} \mathrm{~A}^{-1}$. We have carried out all these calculations in counts rather than calibrated flux units in order to stay closer to basic count statistics. This advantage offsets the slight inaccuracy of converting counts into fluxes for the entire spectral window.

\section{Analysis and discussion}

\subsection{Correction to the upper limit}

We need first to correct our upper limit flux from any absorption that would not take place in the object but 
rather along its line of sight in the intergalactic medium or in our Galaxy. At the low redshift of Mrk 54 the intergalactic medium opacity can be neglected. As to the gas-phase absorption in our Galaxy, we have seen that the choice of our $16 \AA$ spectral window makes significant absorption by neutral hydrogen unlikely. Most of the known interstellar metal lines, especially $\mathrm{O}$, are also found to be avoided and the equivalent width of the corresponding absorption is negligible with respect to the width of the window. For the molecular hydrogen that FUSE has shown to be present along most of the extragalactic lines of sight (Shull et al. 2000) we have searched for the presence of the most significant $R(0)$ and $R(1)$ lines of the low-rotational levels of the Lyman and Werner bands, following the identifications in the line of sight of ESO 141-055 (Shull et al. 2000) and the tables of Barnstedt et al. (2000). We found no clear identifications even in the brightest parts of the spectrum. By comparison with the examples of ESO 141055 and Mrk 876 (Shull et al. 2000) we conclude that $N\left(\mathrm{H}_{2}\right)<1 \times 10^{18} \mathrm{~cm}^{-2}$ in the direction of Mrk 54. A difficulty of this comparison comes from the fact that the spectra of ESO 141-055 and Mrk 876 have better signal to noise ratio than Mrk 54 (the objects are approximately two times brighter and the exposure times two times longer). We have used the on-line spectral simulator to explore how the detection is affected by these differences and found that a $\mathrm{H}_{2}$ column density of $1 \times 10^{18} \mathrm{~cm}^{-2}$ would still have been detected in the conditions of our Mrk 54 spectrum. If we give up any comparison with observed spectra and use the simulator alone, a lower limit of $1 \times 10^{17} \mathrm{~cm}^{-2}$ is found. We have then used the simulator to directly calculate the fraction of energy that such $\mathrm{H}_{2}$ column densities would absorb into our specific window of $16 \AA$; we found a fraction of 0.015 for $1 \times 10^{17} \mathrm{~cm}^{-2}$ and 0.043 for the more conservative limit of $1 \times 10^{18} \mathrm{~cm}^{-2}$. We have therefore applied no correction for $\mathrm{H}_{2}$ absorption to our upper limit on the LyC of Mrk 54. This case may well be a fortunate circumstance given the galactic $\mathrm{H}_{2}$ column densities currently reported for extragalactic lines of sight (Shull et al. 2000; Vidal-Madjar et al. 2000). In the absence of a control of the amount of $\mathrm{H}_{2}$ along the line of sight, that FUSE now offers, previous measurements may well have been affected by this problem. If accounted for, the $\mathrm{H}_{2}$ contamination would have led to even less restrictive limits on the LyC escape fraction than established by Hurwitz et al. (1997).

Lastly, the Galactic dust extinction is a more severe and more uncertain factor. In contrast to the thinking for the past 17 years, it is very likely that a residual extinction is present at high Galactic latitudes, even in the directions with the lowest H I column densities (Schlegel et al. 1998). For Mrk 54 we have adopted $E(B-V)=0.015$, as given by the NASA Extragalactic Database on the basis of this reference. This color excess is consistent with a low fraction of hydrogen in the molecular state (Savage et al. 1977) and therefore with previous limits placed on the $\mathrm{H}_{2}$ column density along the line of sight to Mrk 54. This results in a foreground extinction of $0.29 \mathrm{mag}$ in our far-UV window (centered at $\sim 940 \AA$ ) using an extrapolation of the parameterized extinction law of Cardelli et al. (1989) shortward of $1000 \AA$. Such an extrapolation is supported by the measurements of Buss et al. (1994). We therefore have to account for a factor of 1.306 absorption that does not occur in Mrk 54; our upper limit on the $f(900)$ flux of Mrk 54 is increased to $6.15 \times 10^{-16} \mathrm{erg} \mathrm{cm}^{-2} \mathrm{~s}^{-1} \mathrm{~A}^{-1}$.

\subsection{Evaluation of the $L y C$ escape fraction}

We can now proceed with the calculation of an upper limit on the $\mathrm{LyC}$ escape fraction $f_{\text {esc }}$ defined as the fraction of emitted $900 \AA$ photons that escapes the galaxy without being absorbed by interstellar material. We need first to have a relation between the $f(900)$ flux and the total number of LyC photons since it is this latter quantity that can be physically constrained by the observed $\mathrm{H} \alpha$ flux. Leitherer et al. (1995) have shown that there is a narrow relation, relatively independent of star formation histories and initial mass functions, between the luminosity $L(900)$ at about $900 \AA$ and the total number $N_{\text {Ly }}$ of LyC photons of a burst population: $\log \left(N_{\mathrm{Ly}} / L(900)\right)=$ $13.28 \pm 0.16$ (photons $\AA \operatorname{erg}^{-1}$ ).

Assuming 0.45 as the number of $\mathrm{H} \alpha$ photons per recombination (case $B$ recombination at $10^{4} \mathrm{~K}$ ), we write the number of LyC photons $N_{\mathrm{Ly}}$, corrected by the fraction of those escaping photoelectric absorption $(f 1)$ or trapped by dust before ionization $(f 2)$,

$N_{\mathrm{Ly}}(1-f 1-f 2)=7.34 \times 10^{11} 4 \pi D^{2} f(\mathrm{H} \alpha) 10^{0.4 A(\mathrm{H} \alpha)}$

with $f(\mathrm{H} \alpha)$ the observed $\mathrm{H} \alpha$ flux (in erg $\mathrm{cm}^{-2} \mathrm{~s}^{-1}$ ), $A(\mathrm{H} \alpha)$ the extinction at $\mathrm{H} \alpha$ in the observed galaxy and $D$ the distance (in $\mathrm{cm})$. The luminosity $L(900)$ is

$L(900) f_{\mathrm{esc}}=4 \pi D^{2} f(900)$.

The continuum flux $f(900)$ is corrected for gas-phase absorption in the Galaxy and foreground dust extinction. It should be noted that $f_{\text {esc }}$ may be different from $f 1$ because of photons escaping photoelectric absorption but absorbed by dust and the frequency dependence of photoelectric absorption. Combination of the above equations gives:

$f_{\mathrm{esc}}=\frac{26 f(900) 10^{-0.4 A(\mathrm{H} \alpha)}(1-f 1-f 2)}{f(\mathrm{H} \alpha)}$.

As we are dealing with an upper limit calculation in the case of Mrk 54 and we expect $f_{\text {esc }}, f 1$, and $f 2$ to be small it makes sense to write

$f_{\mathrm{esc}} \leq \frac{26 f(900) 10^{-0.4 A(\mathrm{H} \alpha)}}{f(\mathrm{H} \alpha)}$

An $\mathrm{H} \alpha$ flux of $2.6 \times 10^{-13} \mathrm{erg} \mathrm{cm}^{-2} \mathrm{~s}^{-1}$ has been obtained for Mrk 54 from spectrophotometric observations (Boselli 2000). An extinction $A(\mathrm{H} \alpha)=0$ (although a value of 0.35 was obtained from the Balmer decrement measured along one slit position) has been adopted in order to account for 
the uncertainty on the $\mathrm{H} \alpha$ flux in the upper limit calculation of Eq. (2). We finally obtain $f_{\text {esc }}<0.062$ for Mrk 54 .

This result adds another significant limit to three other cases with limits of $3.2 \%, 5.2 \%$ and $11 \%$ as obtained with HUT and the re-analysis by Hurwitz et al. (1997) (Mrk 66 with a limit of only $57 \%$ is left over from this comparison). All the values obtained so far are fully compatible with current estimates in the range $2 \%-10 \%$ based either on theoretical models (Dove \& Shull 1994; Dove et al. 2000) or the implications of $\mathrm{H} \alpha$ observations of the Magellanic Stream (Bland-Hawthorn \& Maloney 1999) or NGC 3067 (Tumlinson et al. 1999).

\subsection{Neutral hydrogen}

Neutral hydrogen is expected in galaxies where active star formation is taking place and its impact on the escape of ionizing photons is thought to depend heavily on topology. In Mrk 54 the neutral hydrogen is directly seen along the line of sight as Lyman series absorption lines (down to Ly7) at the redshift of the parent galaxy. These features are accompanied by a number of metal absorptionlines with different possible origins (interstellar gas, stellar photospheres and stellar winds) as currently found in starburst galaxies (e.g. Heckman et al. 1998 and references therein). Most of the analyses so far have concentrated on the features (including $\mathrm{Ly} \alpha$ ) accessible with $I U E$ or the spectrographs of the HST. To the best of our knowledge, the discussion of features blueward of Ly $\alpha$ have been limited to the $\mathrm{O}$ VI $+\mathrm{Ly} \beta+\mathrm{C}$ II profile in the four starbursts observed with HUT (Gonzalez-Delgado et al. 1997, 1998).

Leaving a full discussion of absorption features in Mrk 54 to another context, we have only tried to interpret the H I Lyman series absorption profiles. Within the uncertainties of background subtraction, the lines appear almost black at their centers (this is less clear with the $\mathrm{Ly} \beta$ and $\mathrm{Ly} \gamma$ lines than with the three lines displayed in Fig. 1) and indicate a large covering fraction of neutral hydrogen. The determination of the neutral hydrogen column density implied by the absorption profiles is however difficult because of the low signal-to-noise ratio and the possible blending with galactic interstellar lines (not to speak of nightglow emission lines) resulting in very uncertain continuum levels: the profile fitting allows column densities of the order of $10^{21} \mathrm{~cm}^{-2}$ with a low $b$ Doppler parameter as well as low column densities with $b$ up to $\sim 300 \mathrm{~km} \mathrm{~s}^{-1}$. Large velocity spreads have already been reported in nearby and high redshift starburst galaxies (e.g. Gonzalez-Delgado et al. 1998; Pettini et al. 2000).

This finally leads us to place the discussion in the context of the large-scale outflows that have been revealed in the interstellar media of starburst galaxies (e.g. Heckman 2000 and references therein) and play a role in the escape of Ly $\alpha$ emission (e.g. Kunth et al. 1998). As the Ly $\alpha$ emission often appears redshifted with respect to absorption features, the simplest interpretation is that the only Ly $\alpha$ photons that escape unabsorbed are those backscattered from the far side of the outflow, whereas the approaching part is seen in absorption against the stellar continuum (Pettini et al. 2000). In this picture, the LyC photons cannot escape in the same way as the backscattered Ly $\alpha$ photons but it is likely that the galactic winds generate holes in the $\mathrm{H}$ I distribution through which LyC photons can also escape in different directions. A more complete model, including time evolution effects and accounting for the variety of Ly $\alpha$ absorption and emission profiles observed by Kunth et al. (1998), has been developed by Tenorio-Tagle et al. (1999); in the phase where the conical $\mathrm{H}$ II region extends to the galaxy outer edge, LyC and Ly $\alpha$ photons should escape in the same direction. With only upper limits obtained so far, and only five nearby galaxies, it is difficult to constrain one particular model by examining how the LyC escape fraction correlates with the Ly $\alpha$ escape and the blend of absorption and emission at Ly $\alpha$; for instance the two objects with the tightest LyC escape fraction upper limit (Mrk 496 and IRAS 08339) both have Ly $\alpha$ in emission. The only sure conclusion of this discussion is the role of anisotropies that act to increase the randomness of the $\mathrm{LyC}$ escape fraction for the observer.

\section{Comparison with the Lyman break galaxies}

The upper limits $(<10 \%)$ obtained at $z \approx 0$ are in stark contrast with a LyC escape fraction larger than $50 \%$ reported by Steidel et al. (2001) from the detection of the LyC radiation in a composite spectrum of Lyman break galaxies at $z=3.4$. In Steidel et al. the LyC escape fraction is normalized by the fraction of $1500 \AA$ photons that escapes. The surprisingly large value $(>50 \%)$ results from the fact that the $f(1500) / f(900)$ flux ratio is, after the necessary correction for the intergalactic medium opacity at high redshifts, close to that predicted from spectral synthesis models without any LyC self-absorption from neutral hydrogen in the galaxy. The escape fractions used at low and high redshifts are therefore different both in reference wavelength (the escape fraction for nearby galaxies refers to $\mathrm{H} \alpha$ vs. $1500 \AA$ for the Lyman break galaxies) and in the treatment of dust extinction (dust extinction is accounted for at $\mathrm{H} \alpha$ in nearby galaxies, whereas it is not for the Lyman break galaxies). Before trying to quantify this difference between the escape fractions, we have first examined a quantity that is directly observed in both cases, the flux ratio $f(1500) / f(900)$.

\subsection{The $f(1500) / f(900)$ flux ratio of nearby galaxies}

We have calculated the $f(1500) / f(900)$ flux ratios in Mrk 54 and the four nearby star-forming galaxies observed with HUT (Table 1). For the comparison with the high- $z$ galaxies to be meaningful we have to use the $f(900)$ upper limit fluxes corrected for gas-phase absorption and foreground dust extinction. As for Mrk 54, the latter correction has been based on the $E(B-V)$ of Schlegel et al. (1998) given in the NED. These corrections are slightly 
different from those of Leitherer et al. (1995) but the conclusions are not changed. All steps of calculations are detailed in the notes to Table 1 . The $f(1500) / f(900)$ flux ratios of the nearby objects (Table $1 \mathrm{Col} .7$ ) are found to be larger than the value obtained by Steidel et al. (2001) from their composite spectrum of Lyman-break galaxies (note that their value of 4.6 translates into a value of 1.7 in the units of Table 1). This is especially true for Mrk 54, essentially because no correction for $\mathrm{H}$ I absorption has to be applied.

Although the escape of LyC photons is probably highly variable from galaxy to galaxy, the number of nearby objects is sufficient to suggest that the escape is easier at redshift $\approx 3.4$ than at low redshift. This trend is now supported by similarly large $f(1500) / f(900)$ flux ratios recently reported by Ferguson (2001) for seven galaxies at $z \approx 1$ in the HDF. The UV luminosity may also be a factor in the sense that higher luminosity at $\approx 1500 \AA$ would imply more photons for ionizing the gas and possibly easier escape. The nearby galaxies have UV luminosities ranging from $7.2 \times 10^{28} \mathrm{erg} \mathrm{s}^{-1} \mathrm{~Hz}^{-1}$ at $\sim 1500 \AA$ for Mrk 54 to ten times less. However, this largest luminosity is similar to the demagnified luminosity of cB58 (Pettini et al. 2000) reported by Steidel et al. as representative of the galaxies chosen for the composite spectrum.

\subsection{The $f(1500) / f(900)$ flux ratio of Ly break galaxies}

We have also examined the $f(1500) / f(900)$ flux ratio obtained for the Lyman break galaxies by Steidel et al. after correction for the IGM opacity. This ratio implies an escape fraction (when normalized to $1500 \AA$ ) $\geq 0.5$. A smaller escape fraction in the absolute sense might be obtained if only a fraction of the $1500 \AA$ photons escape without being absorbed by dust. This argument is given by Steidel et al. and used by Loeb \& Barkana (2000) to infer an escape fraction of $\sim 10 \%$, close to the limits obtained for nearby galaxies. There is, however, a serious limitation with this explanation: a significant dust extinction at $1500 \AA$ would likely imply a larger dust extinction at $900 \AA$ and in turn a smaller intrinsic $f(1500) / f(900)$ ratio, uncomfortably small with respect to those given by stellar population synthesis models (e.g. Bruzual \& Charlot 1993; Charlot 1996; Leitherer et al. 1999). The flatness (in $f_{\nu}$ ) of the composite spectrum obtained by Steidel et al. also suggests a low extinction according to the correlation between the ultraviolet extinction and the slope $\beta$ of the far-UV spectrum (Calzetti et al. 1994). The result of Steidel et al. is puzzling in another respect. If the escape of LyC photons is anisotropic as discussed above and suggested by Pettini et al. (2000), it would be difficult to have only the cases with favorable directions appear in a composite spectrum. It is also true that the galaxies contributing to the composite spectrum have been selected using criteria that would favor the least extinguished and perhaps lowest covering fraction.

\subsection{Quantifying the difference between escape fractions}

The LyC escape fraction appears in Eq. (2) as the $f(900)$ flux divided by the $\mathrm{H} \alpha$ flux and is defined by Steidel et al. as the $f(900)$ flux normalized by the fraction of $1500 \AA$ photons that escapes. It is tempting to establish a relation between these two forms of the escape fraction, independent of the $f(900)$ flux. Equation (2) can be written as (neglecting the factors $f 1$ and $f 2$ )

$$
f_{\mathrm{esc}}=26 \frac{f(900)}{f(1500)} \frac{f(1500)}{f(\mathrm{H} \alpha)} 10^{-0.4 A(\mathrm{H} \alpha)} .
$$

On the other hand the escape fraction as defined by Steidel et al. is

$f_{\mathrm{esc} 2} \approx 1.1 \frac{f(900)}{f(1500)}$

with 1.1 being the $f(1500) / f(900)$ ratio (in units of Table 1) as expected from stellar synthesis models and adopted by Steidel et al. From the ratio of the two equations we get

$\frac{f_{\mathrm{esc} 2}}{f_{\mathrm{esc}}} \approx 0.04 \frac{f(\mathrm{H} \alpha)}{f(1500)} 10^{0.4 A(\mathrm{H} \alpha)}$.

The ratio of the two escape fractions appears to depend on the $\mathrm{H} \alpha$ to far-UV (1500 $\AA$ ) ratio, a quantity more currently measured than the flux at $900 \AA$. The latter ratio (expressed in $\AA$ with an UV flux in $\operatorname{erg~cm}^{-2} \mathrm{~s}^{-1} \mathrm{~A}^{-1}$ ) can in principle take any value from near zero (a burst older than 7 Myrs without any ionizing star) to very large (dust extinction). However, it is currently measured in the range 10-40 Å (e.g. Buat et al. 1987; Meurer et al. 1999; Sullivan et al. 2000; Bell \& Kennicutt 2001) for the bright, lowextinction and UV-selected star-forming galaxies, such as we are dealing with in this paper. The calibrations of Kennicutt (1998) for the far-UV and $\mathrm{H} \alpha$ star formation rates correspond to an $\mathrm{H} \alpha$ to far-UV ratio of $13 \AA$ and the model calculations of Glazebrook et al. (1999) encompass the range $6-17 \AA$ (extinction not included). The $f_{\text {esc } 2} / f_{\text {esc }}$ ratio is therefore found in the range $0.4-5$, assuming an extinction at $\mathrm{H} \alpha$ in the range $0-1.2$ (Kennicutt 1998). This ratio can be calculated for the nearby galaxies and indeed falls into the expected range. The large range obtained shows the difficulty of comparing escape fractions with different definitions.

In conclusion, the difference between the large LyC escape fraction reported at high redshift and the current upper limits in nearby galaxies must be interpreted with caution because of the difference of the definitions of the escape fractions used. In contrast, the $f(1500) / f(900)$ flux ratios in nearby star-forming galaxies suggest that the $\mathrm{LyC}$ photons escape, at least in the direction of the observer, less easily from nearby galaxies than from high redshift galaxies. More and better measurements of the Lyman continuum of nearby galaxies are needed to distinguish a possible evolution effect from selection effects. 
Table 1. Calculation of the $f(1500) / f(900)$ ratio in nearby galaxies (the ratio of 4.6 reported by Steidel et al. is 1.7 in the units of the table).

\begin{tabular}{lcccccc}
\hline Object & $f(900)$ & $E(B-V)$ & $f_{\mathrm{c}}(900)$ & $f(1500)$ & $f_{\mathrm{c}}(1500)$ & $f_{\mathrm{c}}(1500) / f_{\mathrm{c}}(900)$ \\
& $(2)$ & $(3)$ & $(4)$ & $(5)$ & $(6)$ & $(7)$ \\
\hline IRAS 08339+6517 & $<1.7 \times 10^{-15}$ & 0.092 & $<8.8 \times 10^{-15}$ & $2.8 \times 10^{-14}$ & $5.6 \times 10^{-14}$ & $>6.4$ \\
Mrk 1267 & $<1.4 \times 10^{-15}$ & 0.034 & $<2.6 \times 10^{-15}$ & $8.5 \times 10^{-15}$ & $1.1 \times 10^{-14}$ & $>4.2$ \\
Mrk 66 & $<1.6 \times 10^{-15}$ & 0.012 & $<2.0 \times 10^{-15}$ & $7.5 \times 10^{-15}$ & $8.2 \times 10^{-15}$ & $>4.1$ \\
Mrk 496 & $<1.6 \times 10^{-15}$ & 0.020 & $<2.3 \times 10^{-15}$ & $8.0 \times 10^{-15}$ & $9.3 \times 10^{-15}$ & $>4.0$ \\
Mrk 54 & $<4.7 \times 10^{-16}$ & 0.015 & $<6.2 \times 10^{-16}$ & $2.2 \times 10^{-14}$ & $2.5 \times 10^{-14}$ & $>40$ \\
\hline
\end{tabular}

Notes: all fluxes are in $\mathrm{erg} \mathrm{cm}^{-2} \mathrm{~s}^{-1} \mathrm{~A}^{-1}$.

Column (2) Corrected for gas-phase absorption (Table 1, Col. 12 of Hurwitz et al. 1997 for the galaxies observed with HUT). Column (3) Foreground extinction from the NASA Extragalactic Database. Column (4) Corrected for foreground extinction with $A=19.36 E(B-V)$, resulting from the extrapolation to $\approx 940 \AA$ of the extinction law of Cardelli et al. (1989). Column (5) From the HUT or IUE spectra (Leitherer et al. 1995; Kinney et al. 1993). Column (6) Corrected for foreground extinction with $A=8.26 E(B-V)$.

\section{Implications}

The stellar contribution to the hydrogen-ionizing background has been investigated by a number of authors and its intensity anticipated as a function of the $\mathrm{LyC}$ escape fraction. These results can therefore be briefly revisited in the light of present data and especially the $f(1500) / f(900)$ ratio.

With calculations similar to those of Madau et al. (1999) and Madau (2000), Steidel et al. directly use their measured $f(1500) / f(900)$ ratio to translate the luminosity function of Lyman break galaxies (at $1500 \AA$ ) into a distribution of Lyman continuum luminosities. They find that the 900- $\AA$ emissivity of galaxies at $z \sim 3$ exceeds the contribution of QSOs by a factor of 5 . This result supports the role of star formation in the early re-ionisation of the hydrogen but relies on the assumption that the measured $f(1500) / f(900)$ ratio is characteristic of the whole population of Lyman-break galaxies.

At low redshifts, Giallongo et al. (1997) and Shull et al. (1999) have shown that the galaxy contribution to the ionizing background rivals that of QSOs for an escape fraction of $5 \%$. Such a value is compatible with the present set of upper limits obtained with nearby starforming galaxies. In the same vein as above, an upper limit on the $900-\AA$ emissivity of galaxies at $z \approx 0$ can be directly translated from the far-UV luminosity density of Sullivan et al. (2000), using the $f(1500) / f(900)$ ratios in Table 1. As the data of Sullivan et al. (2000) are at $2000 \AA$ and a mean redshift of 0.15 , we have first used relations established by Cowie et al. (1999) to get the emissivity $0.9 \times 10^{26} \mathrm{erg} \mathrm{s}^{-1} \mathrm{~Hz}^{-1} \mathrm{Mpc}^{-3}$ at $1500 \AA$ and $z \approx 0$ (this would be $0.7 \times 10^{26}$ with an $(1+z)^{4}$ evolution factor). With the tightest constraints of Mrk 54 in Table 1 we get a $900-\AA$ luminosity density $<8 \times 10^{23} \mathrm{erg} \mathrm{s}^{-1} \mathrm{~Hz}^{-1} \mathrm{Mpc}^{-3}$, which is close to the emissivity due to galaxies evaluated by Shull et al. (1999) for an escape fraction of $5 \%$ that would provide a comparable background to AGN.

All the above calculations raise the issue of whether the value used for the $\mathrm{LyC}$ escape fraction is representative of the whole population of galaxies. Given all the factors (distribution of neutral gas, orientation) that make this parameter random, it appears a crude oversimplification to use a unique value for all galaxies. At the same time, any effort to understand the variations of this parameter should carry the same level of difficulty as establishing the LyC luminosity function itself.

As to the present calculations on Mrk 54 and comparison with other nearby galaxies, it is likely, from the mere fact that the LyC escape fraction is random, that any average value should lie below the current upper limits. In addition, any dust extinction decreases the $\mathrm{LyC}$ escape fraction below the value expected from neutral hydrogen absorption alone (see Eqs. (1) or (2)). The nearby galaxies under study, selected with significant UV flux for the prospect of detecting ionizing radiation, are probably not representative of the whole population in terms of dust extinction. Such an effect would be present when it comes to using an average escape fraction with an $\mathrm{H} \alpha$ luminosity density for deriving the LyC luminosity density of galaxies.

\section{Conclusions}

The wavelength domain of the Far Ultraviolet Spectroscopic Explorer (FUSE) down to $905 \AA$ allows us to observe the $\mathrm{LyC}$ radiation of low redshift galaxies above the limit of photoelectric absorption by neutral hydrogen in our Galaxy. The high spectral resolution of FUSE combined with an appropriate redshift selection allows us to avoid Lyman series absorption by neutral hydrogen in our Galaxy over a reasonably large wavelength range. The high spectral resolution of FUSE 
is also useful to identify (and eventually to correct for) possible gas-phase absorption from molecular hydrogen and species other than hydrogen. The observation of the star-forming galaxy Mrk $54(z=0.0448)$ is reported here with the following results:

(i) The LyC flux is not detected. An upper limit can be reasonably evaluated by comparison with the background in adjacent zones of the detector. It is measured over three spectral windows ( $16 \AA$ in total) that are free, to a high probability, of any absorption by neutral hydrogen in our Galaxy. No correction for molecular hydrogen absorption is needed, according to the lack of the corresponding absorption features in the spectrum at longer wavelengths. A correction for foreground dust extinction is performed.

(ii) By comparison with the $\mathrm{H} \alpha$ flux of Mrk 54, the resulting upper limit on the $\mathrm{LyC}$ flux translates into an upper limit LyC escape fraction $f_{\text {esc }}<0.062$. This result is compatible with current models for the escape of $\mathrm{LyC}$ radiation in galaxies and adds another significant limit to three other cases with limits of the order or less than $10 \%$.

(iii) A low escape of LyC photons is expected in Mrk 54 from the large covering fraction of neutral hydrogen revealed by the Lyman series absorption lines down to Ly7. The randomness of the LyC escape fraction as resulting from the geometry (anisotropy in escape direction, hole in the neutral gas distribution) and large-scale galactic outflows is emphasized. No relation has yet been established between the LyC and the Ly $\alpha$ emission escape in starburst galaxies.

(iv) The contrast between the detection of significant LyC flux in a composite spectrum of Lyman break galaxies (Steidel et al. 2001) at $z \approx 3.4$ and the fact that only upper limits have been obtained in nearby star-forming galaxies is investigated. The $f(1500) / f(900)$ flux ratio is found to be larger in nearby galaxies than in the composite spectrum of Steidel et al. (2001). The possible trend of LyC photons escaping more easily at high redshifts than at low redshifts is now supported by the STIS data at $z \approx 1$ of Ferguson (2001) but more and better observations of nearby galaxies are needed to distinguish an evolution effect from selection and random effects.

(v) In addition to an evaluation from the $\mathrm{H} \alpha$ emissivity and the LyC escape fraction, the $900-\AA$ emissivity of galaxies and potentially the ionizing background radiation can now be derived from the measured UV luminosity density and (UV to $900 \AA$ ) ratio.

Acknowledgements. We thank the FUSE Team at JHU for the successful operation of such a complex instrument. J-M D thanks G. Kriss for providing specific scripts for reducing FUSE data with IRAF, and A. Boselli and G. Gavazzi for their optical spectroscopic observation of Mrk 54. This research has made use of the NASA/IPAC Extragalactic Database (NED) which is operated by the Jet Propulsion Laboratory, California Institute of Technology, under contract with the National Aeronautics and Space Administration.

\section{References}

Barnstedt, J., Gringel, W., Kappelmann, N., \& Grewing, M. 2000, A\&AS, 143, 193

Bechtold, J., Weymann, R. J., Lin, Z., \& Malkan, M. A. 1987, ApJ, 315, 180

Bell, E. F., \& Kennicutt, R. C. 2001, ApJ, 548, 681

Bland-Hawthorn, J., \& Maloney, P. R. 1999, ApJ, 510, L33

Boselli, A. 2000, private communication

Bruzual, A. G., \& Charlot, S. 1993, ApJ, 405, 538

Buat, V., Donas, J., \& Deharveng, J.-M. 1987, A\&A, 185, 33

Buss, R. H., Allen, M., Mc Candliss, S., et al. 1994, ApJ, 430,630

Calzetti, D., Kinney, A. L., \& Storchi-Bergmann, T. 1994, ApJ, 429,582

Cardelli, J. A., Clayton, G. C., \& Mathis, J. S. 1989, ApJ, 345,245

Charlot, S. 1996, From Stars to Galaxies: The Impact of Stellar Physics on Galaxy Evolution, ed. C. Leitherer, U. Fritzevon-Alvensleben, \& J. Huchra, ASP Conf. Ser., 98, 275

Cowie, L. L. 1988, The post-recombination universe; Proceedings of the NATO Advanced Study Institute (Cambridge, UK, Dordrecht, Kluwer Academic Publishers), 1

Cowie, L. L., Songaila, A., \& Barger, A. J. 1999, AJ, 118, 603

Dove, J. B., \& Shull, J. M. 1994, ApJ, 430, 222

Dove, J. B., Shull, J. M., \& Ferrara, A. 2000, ApJ, 531, 846

Feldman, P. D., Sahnow, D. J., Kruk, J. W., Murphy, E. M., \& Moos, H. W. 2000, preprint

Ferguson, H. C. 2001, Proceedings of the ESO/ECF/StScI Workshop on Deep Fields, October 2000 [astro-ph/01011356]

Giallongo, E., Fontana, A., \& Madau, P. 1997, MNRAS, 289,629

Glazebrook, K., Blake, C., Economou, F., Lilly, S., \& Colless, M. 1999, MNRAS, 306, 843

Gonzalez-Delgado, R. M., Leitherer, C., \& Heckman T. 1997, ApJ, 489, 601

Gonzalez-Delgado, R. M., Leitherer, C., Heckman, T., et al. 1998, ApJ, 495, 698

Haardt, F., \& Madau, P. 1996, ApJ, 461, 20

Heckman, T. M., Robert, C., Leitherer, C., Garnett, D. R., \& van der Rydt, F. 1998, ApJ, 503, 646

Heckman, T. M. 2000, preprint [astro-ph/0009075]

Hurwitz, M., Jelinsky, P., \& Dixon, W. V. D. 1997, ApJ, 481, L31

Kennicutt, R. C. 1998, ARA\&A, 36, 189

Kinney, A. L., Bohlin, R. C., Calzetti, D., Panagia, N., \& Wyse, R. F. G. 1993, ApJS, 86, 5

Kunth, D., Mas-Hesse, J. M., Terlevich, E., et al. 1998, A\&A, 334,11

Lockman, F. J., \& Savage, B. D. 1995, ApJS, 97, 1

Loeb, A., \& Barkana, R. 2000, ARA\&A, preprint [astro-ph/0010467]

Leitherer, C., Ferguson, H. C., Heckman, T. M., \& Lowenthal, J. D. 1995, ApJ, 454, L19

Leitherer, C., Schaerer, D., Goldader, J. D., et al. 1999, ApJS, 123,3

Madau, P., \& Shull, J. M. 1996, ApJ, 457, 551

Madau, P., Haardt, F., \& Rees, M. J. 1999, ApJ, 514, 648

Madau, P. 2000, Phil. Trans. Roy. Soc. A, in press [astro-ph/0003096]

Meiksin, A., \& Madau, P. 1993, ApJ, 412, 34 
Meurer, G. R., Heckman, T. M., \& Calzetti, D. 1999, ApJ, Shull, J. M., Tumlinson, J., Jenkins, E. B., et al. 2000, ApJ, 521,64

Miralda-Escudé, J., \& Ostriker, J. P. 1990, ApJ, 350, 1 538, L73

Moos, H. W., Cash, W. C., Cowie, L. L., et al. 2000, ApJ, 538, L1

Songaila, A., Cowie, L. L., \& Lilly, S. J., 1990, ApJ, 348, 371

Pettini, M., Steidel, C. C., Adelberger, K. L., Dickinson, M., Steidel, C. C., Pettini, M., \& Adelberger, K. L. 2001, ApJ, \& Giavalisco, M. 2000, ApJ, 528, 96 546,665

Savage, B. D., Bohlin, R. C., Drake, J. F., \& Budich, W. 1977, Sullivan, M., Treyer, M., Ellis, R. S., et al. 2000, MNRAS, ApJ, 216, 291 312,442

Sahnow, D. J., Moos, H. W., Ake, T. B., et al. 2000, ApJ, Tenorio-Tagle, G., Silich, S. A., Kunth, D., Terlevich, E., \& $538, \mathrm{~L} 7$ Terlevich, R. 1999, MNRAS, 309, 332

Schlegel, D. J., Finkbeiner, D. P., \& Davis, M. 1998, ApJ, Tumlinson, J., Giroux, M. L., Shull, J. M., \& Stocke, J. T. 500, 5 1999, AJ, 118, 2148

Shull, J. M., Roberts, D., Giroux, M. L., Penton, S. V., \& Vidal-Madjar, A., Kunth, D., Lecavelier des Étangs, A., et al. Fardal, M. A. 1999, AJ, 118, 1450 2000, ApJ, 538, L77 